\title{
Rheumatoide Arthritis
}

Die Resultate von Untersuchungen zu Padmed Circosan zeigen, dass in der Behandlung von rheumatoider Arthritis (RA) auch alternative Therapieansätze verfolgt werden können, um Symptome zu lindern und damit die Lebensqualität der Betroffenen zu erhöhen [1]. Die RA, auch bekannt als chronische Polyarthritis, ist die häufigste Form der chronisch-entzündlichen Gelenkerkrankungen und mit einer Prävalenz von 0,5-1\% weltweit stark verbreitet.

Die Ursachen der RA sind bislang nicht vollständig geklärt, ihre Folgen jedoch beeinträchtigen die Lebensqualität der Betroffenen ganz erheblich. Als Ursache

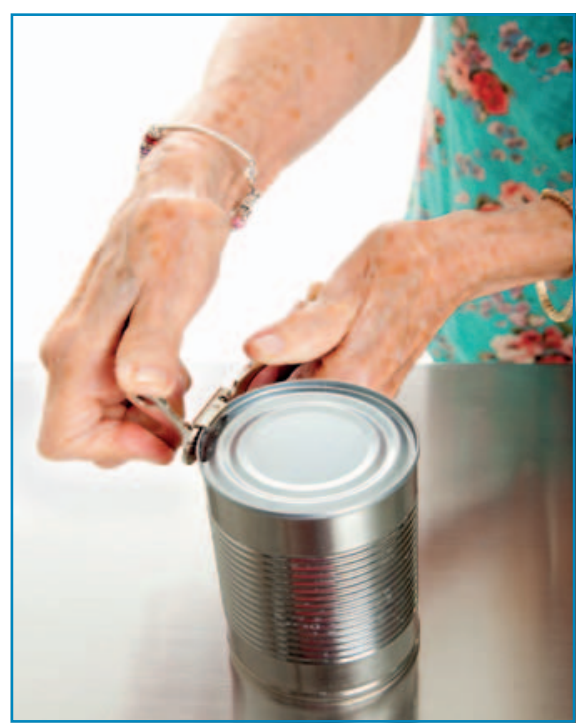

Abb. 1. Ganz alltägliche Bewegungen sind für Betroffene der RA schmerzvoll und beeinträchtigen deren Lebensqualität. wird eine Autoimmunstörung angenommen, bei der körpereigene Substanzen (z.B. Gelenkknorpel) von Immunzellen angegriffen werden. Dabei aktiviert ein Stimulus das angeborene Immunsystem, dendritische Zellen wandern in die Lymphknoten ein und aktivieren über Antigen-Präsentation T-Zellen, was schlussendlich zur Produktion von proinflammatorischen Zytokinen, u.a. TNF- $\alpha$, führt. TNF- $\alpha$ ist ein zentraler Bestandteil in der Entzündungskaskade und stimuliert neben der Produktion von proinflammatorischen Mediatoren auch die Rekrutierung weiterer Immunzellen ins Gelenk. Die Reaktion des Immunsystems wird so in einem positiven Feedbackloop aufrechterhalten, und der Grundstein für ein chronisch-entzündliches Milieu ist gelegt. Erste Symptome einer RA sind gerötete und überwärmte Gelenke. Spätere Folgen reichen von geschwollenen Gelenken bis zu deren Zerstörung durch progressive Knochenerosion und können ganz alltägliche Bewegungen sehr schmerzvoll werden lassen.

Padmed Circosan wird in der Schweiz nach einer Rezeptur der Traditionellen Tibetischen Medizin hergestellt. Es ist ein Vielstoffgemisch und besteht aus 20 pflanzlichen und einem mineralischen Wirkstoff sowie natürlichem Campher. Es wirkt gegen Durchblutungsstörungen mit Beschwerden wie Kribbeln, Einschlafen von Händen und Füssen und Wadenkrämpfen. Verschiedene Studien zeigen weitere mögliche Einsatzgebiete des Präparates, z.B. bei chronischer Pulpitis, Hepatitis B, Atemwegsinfektionen, Multipler Sklerose und RA [2]. All diesen Indikati- onsgebieten gemeinsam sind die zugrundeliegenden chronisch-entzündlichen und Autoimmun-Prozesse. Neben antiinflammatorischen und antioxidativen Eigenschaften konnten auch immunoregulatorische Effekte dieses Vielstoffgemisches gezeigt werden [2]. Eine ältere klinische Studie zur Anwendung von Padmed Circosan bei RA zeigte im Weiteren eine signifikante Abnahme der Ausprägung von Symptomen wie geschwollene Gelenke, Schmerzintensität und Morgensteifigkeit der Gelenke (Dosiserung: 6 Kapseln/Tag über 6 Monate) [3]. In einer experimentellen Studie konnte ausserdem eine hemmende Wirkung von Padmed Circosan auf die induzierte Produktion von TNF- $\alpha$ gezeigt werden [4].

Das pflanzliche Arzneimittel Padmed Circosan kann mit seinen antiinflammatorischen Eigenschaften helfen, Symptome zu lindern und damit die Lebensqualität der Betroffenen zu erhöhen.

\section{Referenzen}

1 Aktuelle Fachinformation PADMED CIRCOSAN ${ }^{\circledR}$ (identisch mit PADMA ${ }^{\circledR} 28$ )

2 Vennos C, et al.: Forsch Komplementmed 2013; 20(suppl 2):25-30.

3 Bernacka K, et al.: 4th Interscience World Conference on Inflammation, Geneva/Switzerland, 15-18 April 1991.

4 Barak V, et al.: Eur Cytokine Netw 2004;15:203-209.

Weitere Informationen bei

PADMA AG

Christian Loepfe

Unterfeldstrasse 1, 8340 Hinwil,

SCHWEIZ

Tel. +41 43-3434444, Fax -888 2626

c.loepfe@padma.ch / www.padma.ch

\section{Donate A Plate ${ }^{\circledR}$ \\ Pipilotti Rist - mit Kunst gegen Brustkrebs \\ Es ist wieder soweit - bunte Schalen schmücken ab Oktober wieder Wartezimmer, Lobbys und Wohnräume! Donate A Plate ist eine nationa- le Solidaritätsaktion, die mit Porzellanschalen ein farbiges Zeichen für Frauen mit Brustkrebs setzt. Dieses Jahr unterstützen hochkarätige Künstlerinnen das Projekt, unter anderem Pipilotti Rist, weltweit eine der bekanntesten zeitgenössischen Videokünstlerinnen. \\ Zeigen Sie Ihr Engagement - machen Sie mit!}

Die Schalen sind nicht nur schön, sondern haben einen besonderen Zweck: Der Erlös von 15 Franken pro Schale kommt der Schweizer Brustkrebshilfe zugute. Bei der Spendenaktion wirken Unternehmen, Spitäler und Privatpersonen mit.
Im Uhrzeigersinn:

Anna Meier, Claudia Caviezel,

Chrissy Angliker, Pipilotti Rist

Bestellen Sie noch heute eine Schale für Ihre Praxis. Sie erhalten zudem Flyer zur Weitergabe an Ihre Patientinnen.

Preis pro Schale:

CHF 44,- exkl. Versandkosten

Tel. +4144227 1131

order@donateaplate.ch www.donateaplate.ch

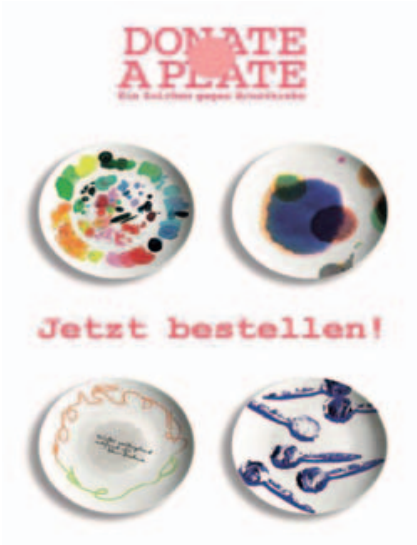

\section{KARGER}

Fax +497614520714 Information@Karger.co www.karger.com
○ 2014 S. Karger GmbH, Freiburg 